\title{
1D crossed gratings for narrow band polarization insensitive reflective filtering
}

\author{
K. Sharshavina1, S. Pelloquin' ${ }^{1}$, E. Daran¹, J.B. Doucet ${ }^{1}$, F. Lemarchand ${ }^{2}$, T. Bégou ${ }^{2}$, J. Lumeau ${ }^{2}$, \\ A. Monmayrant ${ }^{1}$, P. Arguel ${ }^{1}$, O. Gauthier-Lafaye ${ }^{1}$, E. Popov ${ }^{2}$ and A.L Fehrembach ${ }^{2}$ \\ 1. LAAS-CNRS, Université de Toulouse, CNRS, Toulouse, France \\ 2. Aix Marseille Univ, CNRS, Centrale Marseille, Institut Fresnel, F-13013 Marseille, France \\ e-mail: olivier.gauthier-lafaye@laas.fr
}

\begin{abstract}
We present experimental characterizations of 1D crossed Guided Mode Resonant Filters (GMRFs) in the near infrared fabricated by nanoimprint lithography. We will show that subwavelength gratings up to 4" diameter can be fabricated using such techniques. We compare the performances of the GMRFs to the performances of FabryPerot filters and discuss the sensitivity to fabrication inhomogeneity of these components.
\end{abstract}

Keywords: Fabry-Perot filters, GMRF filtersd, sub-wavelength filters.

\section{INTRODUCTION}

Guided Mode Resonance Filters (GMRF) are a new generation of narrowband filters that can be used for embedded spatial spectroscopy. They consist of a subwavelength grating on the top of a slab waveguide. The incident wave is diffracted by the grating. When a diffracted mode matches a guided mode, the incident wave is coupled into the waveguide, and a resonance occurs. In this case the coupled wave slowly leaks out (decouples) as it propagates in the perturbed waveguide. The constructive interference between the decoupled wave and the wave transmitted and reflected non-resonantly generates the filter response. One of their most striking feature is that ultra-narrow band filtering of light can be achieved with a low number of optical layers as compared to Fabry-Perot filters [1]. In this paper we compare experimentally ultra-narrow band FP and GMRF filters and analyse their performances in terms of fabrication homogeneity.

\section{FILTERS FABRICATION}

Narrow band GMRF filters in the $1.55 \mu \mathrm{m}$ range were designed using an optimization algorithm (Clustering Global Optimization) combined with a Fourier Modal Method numerical code. An 8 layer waveguide with antireflective properties was deposited using the Plasma Assisted Reactive Magnetron Sputtering (PARMS) technique. Such deposition method allows extremely high control of the deposition thickness and composition with high layer uniformity over the wafer surface.

After deposition, the $875 \mathrm{~nm}$ period grating is realised using a dedicated soft-mold nanolithography process to realise an etching mask in a resist and afterward etched into the top silica layer. Figure 1 shows a picture of one of the fabricated 1D GMRF filter. Physical characterization of the fabricated GMRF with AFM and Littrow measurements shows a good homogeneity of the fabricated grating. The complete processing method will be detailed during the presentation.

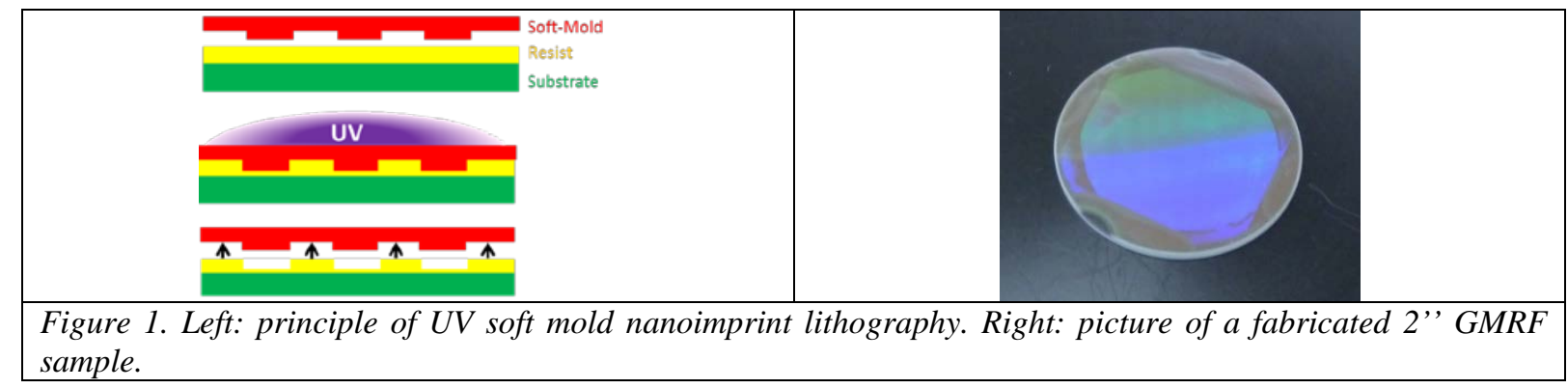

The same deposition technique was used to fabricate Fabry-Perot filters using the same materials. We fabricated 23 layers FP filters in order to theoretically achieve the same linewidth performances as the designed GMRF filters.

\section{FILTERS OPTICAL CHARACTERIZATION}


The filters were characterized on a dedicated test bench using a collimated $1.55 \mu \mathrm{m}$ tunable laser. Figure 2 shows the experimental and theoretical transmission spectra of the FP and GMRF filters. The experimental response of the filters was measured for different points of incidence on the filters.

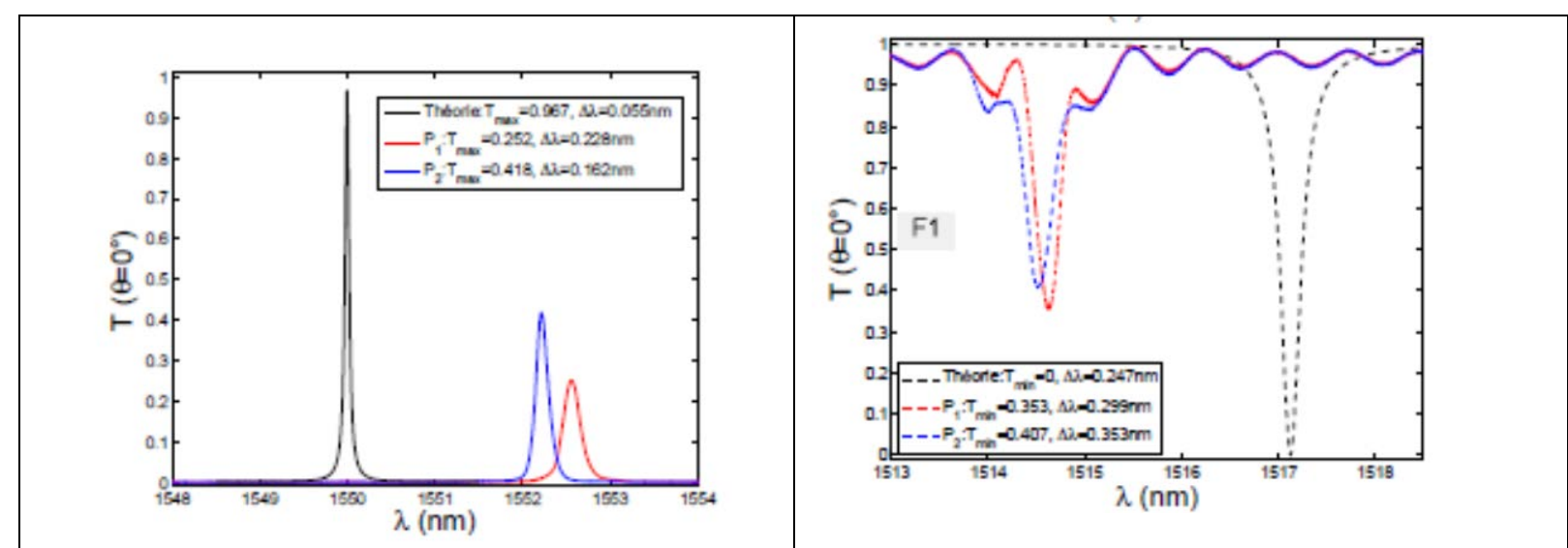

Figure 2. Transmission spectra of the fabricated Fabry-Perot (Left) and GMRF (Right) samples at normal incidence. Black curves are theoretical spectra, blue and red curves are experimental spectra on 2 different positions of the sample.

As seen, the two samples achieve relatively similar performances however far from the expected theoretical performances. We analyse these discrepancies in view of the process inhomogeneities and experimental conditions (with special emphasis on the beam size or angular tolerance of the filters). Such analysis leads to novel design criteria for the optimization of ultra-narrow band GMRF filters.

\section{ACKNOWLEDGEMENTS}

Authors acknowledge support from Centre National d'Etudes Spatiales (CNES) under supervision of J. Loisel.

\section{REFERENCES}

[1] S. S. Wang and R. Magnusson, "Theory and applications of guided-mode resonance filters," Appl. Opt., vol. 32, no. 14, pp. 2606-2613, May 1993. 